\title{
Height augmentation in 11ß-hydroxylase deficiency congenital adrenal hyperplasia
}

Munier A Nour ${ }^{1,4 *}$ and Danièle Pacaud ${ }^{2,3}$

\begin{abstract}
Context: $11 \beta$-hydroxylase deficiency is the second most common form of congenital adrenal hyperplasia. Untreated, this enzyme deficiency leads to virilization, hypertension, and significant height impairment.

Patient: We describe a patient from abroad who first presented to us at age 7 years for follow-up of ambiguous genitalia. He had been investigated and treated in Pakistan at 3-years-of-age following presentation for bilateral cryptorchidism. He was found to have 46, XX karyotype, elevated 17-OH progesterone and was diagnosed with congenital adrenal hyperplasia. In Pakistan, the patient had abdominal hysterectomy, bilateral salpingoophrectomy, and was started on corticosteroid replacement. At 7 years, shortly after immigrating to Canada, height was $138 \mathrm{~cm}$ and BMI $19.3 \mathrm{~kg} / \mathrm{m}^{2}$ (+2.9 SDS and +1.7 SDS, respectively, male growth chart) and blood pressure was greater than the 99th percentile for age and height. The patient had Prader stage III - IV genital anatomy. Bone age was significantly advanced, yielding a severely compromised predicted final adult height. Biochemical evaluation was consistent with $11 \beta$-hydroxylase deficiency congenital adrenal hyperplasia.
\end{abstract}

Intervention and outcome: In an attempt to improve final height, in addition to glucocorticoid replacement, this patient was treated with recombinant growth hormone and a third generation aromatase inhibitor (Letrozole) with an improvement in final height attained as compared with predicted height.

Conclusions: This case of a 46,XX patient raised as male with congenital adrenal hyperplasia due to $11 \beta$-hydroxylase deficiency highlights a number of unique and difficult treatment challenges; specifically, the role of new therapeutic options for optimization of growth in the context of prior suboptimal disease management.

Keywords: Congenital Adrenal Hyperplasia, Growth Hormone, Aromatase Inhibitor, Growth, Steroid 11-beta-Hydroxylase

\section{Introduction}

Congenital adrenal hyperplasia (CAH) due to $11 \beta$ hydroxylase $(11 \beta-\mathrm{OH})$ deficiency is the second most common cause of $\mathrm{CAH}[1]$. It accounts for approximately $5-8 \%$ of all $\mathrm{CAH}$ cases and is caused by mutations in the CYP11B1 gene [2]. The deficiency in $11 \beta-\mathrm{OH}$ results in a buildup of 11-deoxycortisol and 11-deoxycorticosterone. Clinical manifestations of $\mathrm{CAH}$ due to $11 \beta-\mathrm{OH}$ deficiency include virilization and ambiguous genitalia in females. The primary distinguishing feature between the more common 21-hydroxylase $(21-\mathrm{OH})$ deficiency and $11 \beta-\mathrm{OH}$ deficiency

\footnotetext{
* Correspondence: munier.nour@usask.ca

${ }^{1}$ Department of Pediatrics, College of Medicine, University of Saskatchewan, Saskatoon, Saskatchewan, Canada

${ }^{4}$ Department of Pediatrics, College of Medicine, Royal University Hospital, 103 Hospital Drive, Saskatoon, SK S7N OW8, Canada

Full list of author information is available at the end of the article
}

is the presence of hypertension and hypokalemia in the latter.

Similar to $\mathrm{CAH}$ due to 21-OH deficiency, optimization of height in $\mathrm{CAH}$ due to $11 \beta-\mathrm{OH}$ deficiency remains a difficult balance between suppressing adrenal androgen production with corticosteroids and minimizing iatrogenic effects of supra-physiologic corticosteroid treatment. As a result, despite optimal treatment final adult height is often impaired [3].

We report a case of a male-identifying, 46, XX, 11 $\beta$ $\mathrm{OH}$ deficiency $\mathrm{CAH}$ patient who presented to our tertiary care center recently after immigration. At the initial visit in our center, he had significantly compromised projected final adult height. In this report we highlight the significant response to height augmenting therapies, namely a combination of growth hormone $(\mathrm{GH})$ and 
aromatase inhibitor (AI), with documented final adult height in our patient.

\section{Methods}

Bone age assessment was independently assessed using the Greulich and Pyle methodology [4]. Predictions of final adult height were calculated use the Bayley and Pinneau method [5]. Laboratory assessments were completed through locally available laboratory services.

Patient care was provided through outpatient pediatric endocrinology clinic. All risks and benefits of interventions were discussed with patient and family. Appropriate consents were obtained for publication of this report.

\section{Case presentation}

A 7-year-old male patient of Pakistani descent and consanguineous parents (first cousins) presented with ambiguous genitalia for evaluation to our endocrine clinic following assessment of a younger sister. The younger sister had initially been found to have ambiguous genitalia and hypertension while being evaluated in the emergency department for an intercurrent illness. The family had recently immigrated to Canada. The 7-year-old patient had initially received evaluation and treatment at 3-years-of-age while in Pakistan for bilateral cryptorchidism. At that time he was found to have normal electrolytes, a 46, XX karyotype and an elevated $17-\mathrm{OH}$ progesterone $(28 \mathrm{ng} / \mathrm{mL}$; normal $<1 \mathrm{ng} / \mathrm{mL}$ ). He was diagnosed there with non-salt wasting $\mathrm{CAH}$. The specific enzymatic deficiency was not delineated. The patient underwent an abdominal hysterectomy and bilateral salpingoophrectomy. He was started on corticosteroid replacement prior to moving to North America. According to his parents, follow-up was minimal and no corticosteroid dose adjustment was made between his initial presentation at 3 years and when he was first seen in Canada at 7-years-of-age.

At 7 years, height was $137.5 \mathrm{~cm} \mathrm{(+2.9} \mathrm{S.D} \mathrm{on} \mathrm{male} \mathrm{growth}$ curve) (see Figure 1) and blood pressure was greater than the 99th percentile for age and height. The patient had Prader stage III - IV genital anatomy and Tanner stage 1 pubertal development. Baseline investigations demonstrated elevated 17-OHP, 11-deoxycorticosterone, 11-deoxycortisol, and suppressed plasma renin activity (See Table 1). There was significant bone age advancement to 12 years (female standard [4]; see Table 2), for chronological age of 7 years, yielding a predicted final adult height of $152.6 \mathrm{~cm}$ ( -3.3 S.D. for a male) [4]. Findings were consistent with $11 \beta-\mathrm{OH}$ deficiency $\mathrm{CAH}$.

\section{Intervention \& results}

The patient was treated with glucocorticoids while in Pakistan, however, treatment was suboptimal. On initial visit in Canada corticosteroid replacement was optimized to a replacement dose of approximately $15 \mathrm{mg} / \mathrm{m}^{2} /$ day of hydrocortisone. Doses were adjusted as necessary and increased to approximately $18-20 \mathrm{mg} / \mathrm{m}^{2} /$ day of hydrocortisone on average. This was subsequently changed to $\sim 3.5$ to $4.1 \mathrm{mg} / \mathrm{m}^{2} /$ day of prednisone (using a bioequivalence ratio of 5 , this equates to 17.5 to $20.5 \mathrm{mg} / \mathrm{m}^{2} /$ day of hydrocortisone). Despite this treatment, ongoing bone-age advancement and a slowing growth velocity to $4.2 \mathrm{~cm} /$ year resulted in further diminishment of the projected adult height to $150.4 \mathrm{~cm}(-3.7$ S.D.).

After lengthy multidisciplinary discussions with the family surrounding the ethical, social and ethnic considerations of growth optimization, the decision to attempt growth augmentation was undertaken. GH treatment $(0.05 \mathrm{mg} / \mathrm{kg} /$ day; 6 days per week) was initiated at 8.3 years, which resulted in an increase in growth velocity to $7.8 \mathrm{~cm} /$ year. However, in response to continued bone age advancement, an AI, Letrozole ( $2.5 \mathrm{mg}$ daily), was initiated at 9 years of age.

Blood pressure remained elevated despite glucocorticoid replacement and further treatment with Spironolactone and Amlodipine were required for control. At age 12 the patient had insertion of testicular prosthesis. At age 14 hypospadias repair and release of chordae was performed. In addition, removal of breast tissue was performed at this time as well. The patient was not initiated on testosterone supplementation until completion of growth as endogenous androgen production was deemed to be adequate and to help maximize growth potential. Of note, the circulating adrenal androgens were enough to induce progressive pubertal changes such as axillary, pubic and facial hair growth, voice changes and acne.

The patient had no adverse effects from GH or AI treatment including no evidence of dysglycemia, dyslipidemia, hepatic dysfunction, vertebral malformations, nor evidence of bone fragility. Letrozole and GH treatment were continued until growth velocity was negligible at 13.6 years of age. A final adult height of $167.5 \mathrm{~cm}$ (-1.3 S.D. male chart) was achieved (See Figure 1). This represented an increase of $17.1 \mathrm{~cm}$ (2.6 S.D.) when using female bone age standards or $16.4 \mathrm{~cm}$ (2.3 S.D.) when using male bone age standards from the projected final adult height before introducing $\mathrm{GH}$ and AI treatment (Table 2).

\section{Discussion}

A number of complex treatment issues arise in the management of patients with $\mathrm{CAH}$. Issues such as genderidentity, growth, blood pressure management, surgical correction, and pubertal management are among the clinical difficulties addressed by the multidisciplinary medical team. The presented case illustrates a number of these in a young patient with $\mathrm{CAH}$ due to $11 \beta-\mathrm{OH}$ deficiency. Among these issues, in particular, we highlight the unique aspects of optimizing height in this patient with dramatic improvement in final adult height. 


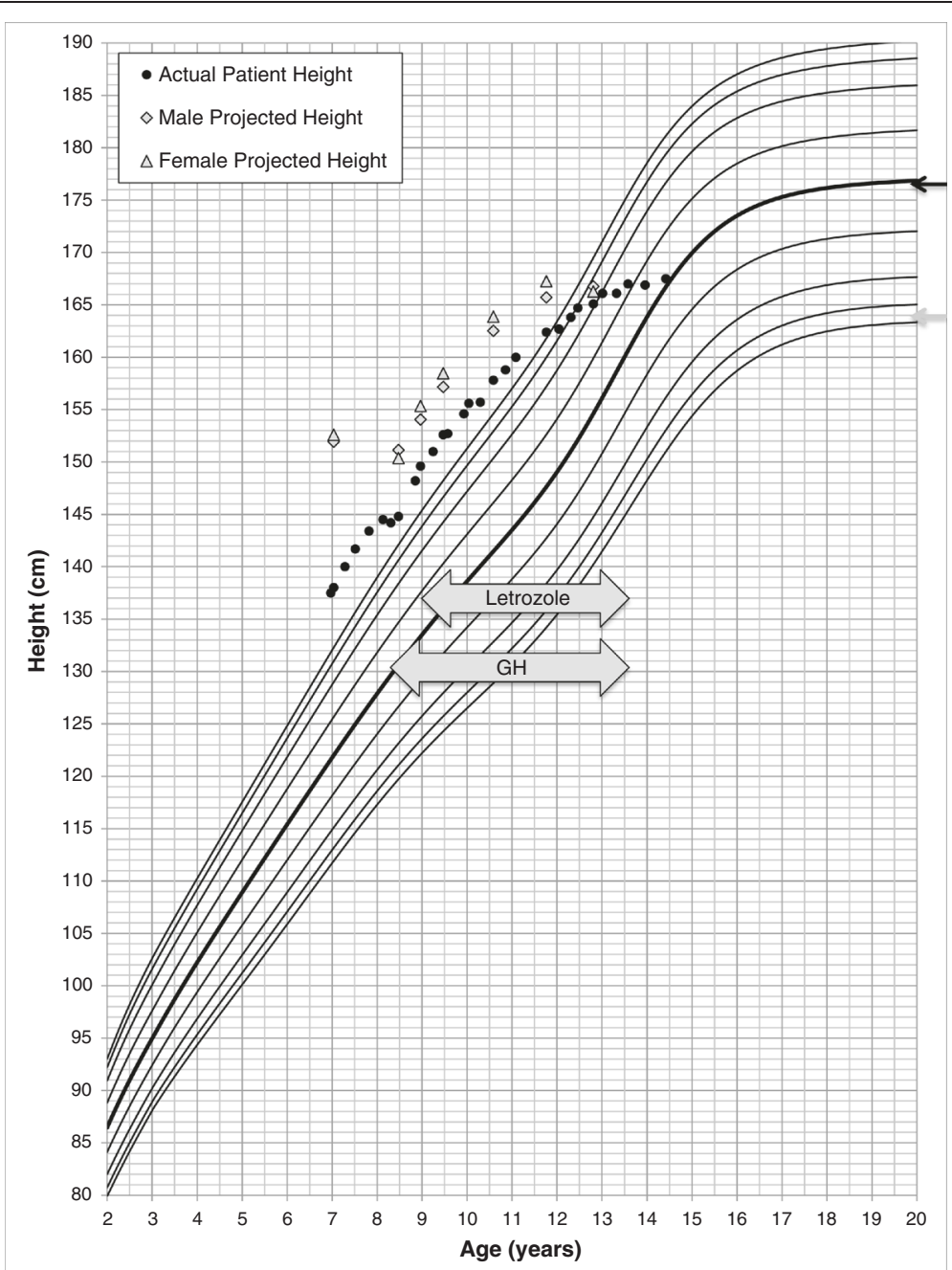

Figure 1 Patient growth chart plotted on male growth chart depicting actual height and calculated predicted height using male (diamond) and female (triangle) reference standards [4,5]. Parental heights are depicted with grey (mother) and black (father) arrows on the right vertical axis. GH and Letrozole treatment duration depicted directly on the chart.

The influence of $\mathrm{CAH}$ due to $11 \beta-\mathrm{OH}$ deficiency on final adult height has not been thoroughly examined in the literature. While no reviews exist in $11 \beta-\mathrm{OH}$ deficiency, a recent systematic review and meta-analysis of $21-\mathrm{OH}$ deficiency CAH demonstrated the final height S.D. score was -1.38 $\left(-1.56\right.$ to $\left.-1.20 ; \mathrm{I}^{2}=90.2 \%\right)$ in patients, despite optimization

Table 1 Laboratory investigations results at time of presentation at 7 years of age

\begin{tabular}{lcc}
\hline Test & Value & Reference range \\
\hline Androstenedione $(\mathrm{nM})$ & 7.7 & $<2.0$ \\
Testosterone $(\mathrm{nM})$ & 2.2 & $<1.0$ \\
17-OH Progesterone $(\mathrm{nM})$ & 30.6 & $<6.0$ \\
11-Deoxycortisol (nM) & 1580 & $<4.5$ \\
11-Deoxycorticosterone (nM) & 40.3 & $<1.03$ \\
Plasma Renin Activity (ng/L/s) & 0.15 & $>0.28$ \\
\hline
\end{tabular}

of CAH treatment [6]. Optimization of height is often a delicate balance between corticosteroid replacement to suppress androgen excess while attempting to limit effects of glucocorticoid excess. The treatment of $11 \beta-\mathrm{OH}$ deficiency involves achieving a similarly challenging balance. Although, as demonstrated with the presented patient, $\mathrm{CAH}$ due to $11 \beta-\mathrm{OH}$ deficiency may require higher glucocorticoid doses than those currently recommended for patients with $\mathrm{CAH}$ due to 21-OH deficiency [7]. Patients with suboptimal control or inadequate treatment, such as our patient, may have substantial reduction in their growth potential [3].

Current Endocrine Society guidelines do not endorse the use of growth augmenting therapies outside of the context of research studies [7]. Our case highlights the success of these treatments in the setting of late diagnosis and initial suboptimal control leading to a significant threat to final height attainment. While we do not 
Table 2 Comparisons of final height estimations by male and female interpretations of bone age radiographs [4,5]

\begin{tabular}{|c|c|c|c|c|c|}
\hline $\begin{array}{l}\text { Chronological } \\
\text { age (years) }\end{array}$ & Height (cm) & Female bone age (years) & Estimated final height $(\mathrm{cm})$ & Male bone age (years) & Estimated final height $(\mathrm{cm})$ \\
\hline 7 & 137.5 & 12 & 152.6 & 14 & 151.9 \\
\hline 8.5 & 144.8 & 13.5 & 150.4 & 15 & 151.1 \\
\hline 9 & 149.6 & 13.5 & 155.3 & 15.5 & 154.1 \\
\hline 9.5 & 152.6 & 13.5 & 158.5 & 15.5 & 157.2 \\
\hline 10.6 & 157.8 & 13.5 & 163.9 & 15.5 & 162.5 \\
\hline 11.8 & 162.4 & 14 & 167.1 & 16 & 165.7 \\
\hline 12.8 & 165.1 & 16 & 166.3 & 17 & 166.8 \\
\hline
\end{tabular}

advocate for such therapies in all CAH patients, in certain dire circumstances their use may be justified. Prior to initiation of GH and Letrozole, our patient had a predicted adult height of 150.4 - $151.1 \mathrm{~cm}$ (depending on female or male reference standard used respectively) which increased by $16.4-17.1 \mathrm{~cm}$ by the time of attainment of final adult height $(167.5 \mathrm{~cm} ;-1.3$ S.D. male standard). This final height is considerably greater than these early predictions and also higher than his genetic calculated mid-parental height for a female of $164 \mathrm{~cm}$ (-1.8 S.D.). He received a total of 5.3 years of GH treatment and 4.6 years of AI treatment. A recent prospective study of $\mathrm{GH}$ use in 21-OH deficiency $\mathrm{CAH}$ found a final height gain of $9.2 \pm 6.7 \mathrm{~cm}$ in males and $10.5 \pm$ $3.7 \mathrm{~cm}$ in females with a mean $\mathrm{GH}$ treatment duration of $5.6 \pm 1.8$ and $4.5 \pm 1.6$ years, respectively [8]. We would advocate that in the setting of extreme impairment of predicted final height, consideration of these therapies may be beneficial. Similar to dosages used in idiopathic short stature, we used a relatively high dose of $\mathrm{GH}$ in our patient given the degree of predicted growth impairment.

Our patient benefited from a combined therapeutic approach using corticosteroids, $\mathrm{GH}$ and aromatase inhibition. Growth hormone treatment was initiated due to ongoing impairments in predicted final adult height despite corticosteroid treatment. While the $\mathrm{GH}$ treatment had benefit in growth velocity $(4.2 \mathrm{~cm} /$ year vs. $7.8 \mathrm{~cm} /$ year respectively) further advancement in bone age continued to compromise projected height benefit. The addition of Letrozole, a third generation steroidal AI, to the treatment regime resulted in dramatic benefit in final height by inducing a slowing bone maturation. Over the span of approximately 2 years there was virtually no advancement of bone maturation.

Aromatase inhibition has been used in an off-label manner in a select group of pediatric conditions, including hyperestrogenism, hyperandrogenism, pubertal gynecomastia and short stature [9]. Their use in CAH has been limited. To date only one study has been conducted examining the use of AIs for height augmentation in $\mathrm{CAH}$ [10]. In this trial, children were randomized to 2 year treatment with 1) Flutamide, testolactone and reduced hydrocortisone dose and fludrocortisone vs. 2) Standard treatment with hydrocortisone and fludrocortisone. The study demonstrated a slight trend to increased predicted final adult height (+0.6 S.D.) in the testolactone/flutamide group, however, the difference was not statistically different. This trial has been ongoing and in 2004, subjects were switched to Letrozole; however, results have not yet been published [11].

The use of AIs in the pediatric setting must be used with caution due to unknown potential long term complications and thus far only modest height benefits. In males treated with AIs, hypertestosteronemia has been observed [12]. This rise in serum testosterone is due to the reduced estrogen feedback on the hypothalamus leading to increased levels of LH. No adverse effects from hypertestosteronemia have been reported. In our patient, previous oophorectomy and female karyotype obviated this risk. Of interest, however, a slight increase in serum androgens was noted with AI introduction. This increase would be possible either through decreased conversion of androgens or an increase in adrenal androgen production. Other potential concerns with AI use have included impaired bone mineral density, body composition and metabolic effects such as dyslipidemias [9]. In addition, there is hesitancy in using AIs in females due to concerns of undesirable effects of hyperandrogenemia. In our patient, these potential effects were viewed as desirable due to his male gender identification. Vertebral malformations have also been reported to be associated with the use of AIs with particular risk when used during the pre-puberty or early puberty phases [13].

Various other growth augmenting therapies have been explored in the setting of $\mathrm{CAH}$ with variable success. In addition to standard therapy with glucocorticoids, these have included growth hormone, GnRH analogs, and antiandrogens to increase final height [14]. In our patient, $\mathrm{GnRH}$ analogs would have been of no benefit given the previous oophorectomy.

Of particular concern for our patient were the cultural and societal implications of height attainment. Being raised male, our patient had societal expectations of a higher final 
height attainment. While evolutionarily this increased final height in males is less drastic than in other species, it remained an important factor for this family when determining treatment goals [15]. The notion that short stature has a negative impact on psychosocial adaptation has been disproved by recent studies done in healthy children [16]. However, in the context of short stature associated with a chronic condition such as $\mathrm{CAH}$, patient and family preferences may need to be considered. In particular, a patient already stigmatized with having a chronic medical condition, gynecomastia, infertility, and abnormal genital anatomy, the normalization of height was an attainable and beneficial goal for patient care.

While assessing bone maturation remains an important tool in monitoring treatment in $\mathrm{CAH}$ [17], considerable difficulty exists in determining the most accurate method to predict final adult height, particularly in patients such as the one presented. Although our patient was raised as male, he had a 46, XX karyotype. The endogenous androgen exposure of our patient was significantly higher than that of an otherwise healthy female. These exposures may have significant influence on bone growth and development. Thus, the interpretation of bone age radiography may be difficult as the differential effect of chromosomal and hormonal influences are ultimately unknown. Interestingly, despite significant variations in bone age interpretation based on gender, the effect on final height prediction for our patient was minimal (see Table 2 and Figure 1). Ultimately, consistency with regard to interpretation of these tests is paramount.

\section{Conclusions}

In summary, we present the growth response of a 46, XX patient with $\mathrm{CAH}$ due to $11 \beta-\mathrm{OH}$ deficiency to final adult height. Despite the significant impairment in predicted adult height due to inadequate initial treatment, this patient demonstrated favorable results with a combined therapeutic approach including the addition of $\mathrm{GH}$ and an $\mathrm{AI}$ to the standard glucocorticoid treatment to optimized growth. Our patient's response to this regimen underscores the need for further research into these new therapeutic avenues.

\section{Competing interests}

The authors declare that they have no competing interests.

\section{Authors' contributions}

MN drafted the initial manuscript. DP provided input and direction for the manuscript and confirmed accuracy of information. Both authors read and approved the final manuscript.

\section{Acknowledgements}

The author's would like to acknowledge the readily available assistance from Mrs. Eileen Pyra.

\section{Author details}

${ }^{1}$ Department of Pediatrics, College of Medicine, University of Saskatchewan, Saskatoon, Saskatchewan, Canada. ${ }^{2}$ Division of Pediatric Endocrinology, Alberta Children's Hospital, Calgary, Alberta, Canada. ${ }^{3}$ Faculty of Medicine,
University of Calgary, Calgary, Alberta, Canada. ${ }^{4}$ Department of Pediatrics, College of Medicine, Royal University Hospital, 103 Hospital Drive, Saskatoon, SK S7N OW8, Canada.

Received: 5 October 2014 Accepted: 23 February 2015

Published online: 15 May 2015

\section{References}

1. Menabò S, Polat S, Baldazzi L, Kulle AE, Holterhus P-M, Grötzinger J, et al. Congenital adrenal hyperplasia due to 11-beta-hydroxylase deficiency: functional consequences of four CYP11B1 mutations. Eur J Hum Genet. 2014;22:610-6.

2. Speiser PW, White PC. Congenital adrenal hyperplasia. N Engl J Med. 2003;349:776-88.

3. Jääskeläinen J, Voutilainen R. Growth of patients with 21-hydroxylase deficiency: an analysis of the factors influencing adult height. Pediatr Res. 1997:41:30-3.

4. Greulich WW, Pyle SI. Radiographic Atlas of Skeletal Development of the Hand and Wrist. 2dth ed. Stanford: Stanford University; 1959.

5. Bayley N, Pinneau SR. Tables for predicting adult height from skeletal age: revised for use with the Greulich-Pyle hand standards. J Pediatr. 1952:40:423-41.

6. Muthusamy K, Elamin MB, Smushkin G, Murad MH, Lampropulos JF, Elamin KB, et al. Adult height in patients with congenital adrenal hyperplasia: a systematic review and metaanalysis. J Clin Endocrinol Metab. 2010;95:4161-72.

7. Speiser PW, Azziz R, Baskin LS, Ghizzoni L, Hensle TW, Merke DP, et al. Congenital adrenal hyperplasia Due to steroid 21-hydroxylase deficiency: an endocrine society clinical practice guideline. J Clin Endocrinol Metab. 2010;95:4133-60.

8. Lin-Su K, Harbison MD, Lekarev O, Vogiatzi MG, New MI. Final adult height in children with congenital adrenal hyperplasia treated with growth hormone. J Clin Endocrinol Metab. 2011;96:1710-7.

9. Wit JM, Hero M, Nunez SB. Aromatase inhibitors in pediatrics. Nat Rev Endocrinol. 2012;8:135-47.

10. Merke DP, Keil MF, Jones JV, Fields J, Hill S, Cutler Jr GB. Flutamide, testolactone, and reduced hydrocortisone dose maintain normal growth velocity and bone maturation despite elevated androgen levels in children with congenital adrenal hyperplasia. J Clin Endocrinol Metab. 2000;85:1114-20.

11. Shulman DI, Francis GL, Palmert MR, Eugster EA. Use of aromatase inhibitors in children and adolescents with disorders of growth and adolescent development. Pediatrics. 2008;121:e975-83.

12. Diaz-Thomas A, Shulman D. Use of aromatase inhibitors in children and adolescents: what's new? Curr Opin Pediatr. 2010;22:501-7.

13. Hero M, Toiviainen-Salo S, Wickman S, Mäkitie O, Dunkel L. Vertebral morphology in aromatase inhibitor-treated males with idiopathic short stature or constitutional delay of puberty. J Bone Miner Res Off J Am Soc Bone Miner Res. 2010;25:1536-43.

14. Javed A, Lteif A, Kumar S. Update on treatment strategies for optimization of final adult height in children with congenital adrenal hyperplasia. Pediatr Endocrinol Rev PER. 2012;10:164-73.

15. Rosenfeld RG. Gender differences in height: an evolutionary perspective. J Pediatr Endocrinol Metab JPEM. 2004;17 Suppl 4:1267-71.

16. Sandberg DE, Bukowski WM, Fung CM, Noll RB. Height and social adjustment: are extremes a cause for concern and action? Pediatrics. 2004; 114:744-50.

17. White PC, Speiser PW. Congenital adrenal hyperplasia due to 21-hydroxylase deficiency. Endocr Rev. 2000;21:245-91. 